\title{
MULTIFREQUENCY OBSERVATIONS OF SYMBIOTIC STARS
}

\author{
Scott J. Kenyon \\ Smithsonian Astrophysical Observatory \\ Harvard-Smithsonian Center for Astrophysics \\ 60 Garden Street \\ Cambridge, MA 02138 USA
}

\begin{abstract}
This paper reviews the discovery of symbiotic stars, and introduces multifrequency observations made during the past two decades.
\end{abstract}

\section{Historical Introduction}

In 1912, Mrs. W.P. Fleming published a remarkable paper entitled "Stars Having Peculiar Spectra" (Fleming 1912). Aside from providing detailed lists of new novae, gaseous nebulae, and O-type stars, Fleming noted several long period variables (including $\mathrm{R}$ Aqr and RW Hya) with bright emission lines and unusually small ranges in brightness. Miss A. Cannon later noted the existence of several $M$ stars with bright $H$ I and He II lines, such as Z And and CI Cyg, in work that went generally unrecognized (Shapley 1922). Merrill and Humason (1932) rediscovered CI Cyg as an anomalous star having bright He II $\lambda 4686$ in combination with the TiO bands characteristic of M4 stars, and noted that RW Hya and AX Per possessed similarly striking features. Merrill and Humason remarked that $\mathrm{T} \mathrm{CrB}$ and $\mathrm{R}$ Aqr also displayed peculiar spectra, and suggested that spectrograms of planetary nebulae and other emission-line stars might be examined for TiO absorption bands. Over a dozen stars with combination spectra had been discovered by 1941 , when Merrill coined the phrase symbiotic stars to describe objects which show evidence for cool M-type photospheric absorption lines and very high temperature emission lines.

Merrill and Humason's short paper produced a great deal of interest in peculiar emission stars, and much effort was spent in obtaining new spectra and monitoring the light variations of these unusual systems. Of special interest were the discoveries by Lindsay (1932) and Greenstein (1937) that AX Pcr and CI Cyg had undergone 2-3 mag nova-like eruptions prior to Merrill and Humason's observations. Searches of the photometric archives revealed similar eruptive activity in $\mathrm{Z}$ And, BF Cyg, and RS Oph, demonstrating that outbursts were normal events for many symbiotic systems (see Figure 1). In addition, a few objects were shown to have quiescent photometric fluctuations which repeated on time scales of 600-900 days (see Payne-Gaposchkin 1957; Kenyon 1986 and references therein). Spectroscopic observations by Merrill at Mt. Wilson Observatory and by Swings and Struve at McDonald Observatory showed that radial velocity variations were correlated with these intensity changes in RW Hya and AG Peg, and lent support to the idea that these objects might be binary systems. Periodic radial velocity variations were not observed in some symbiotics, because their violent eruptive activity made it difficult to follow absorption and emission features from one orbital cycle to the next. 

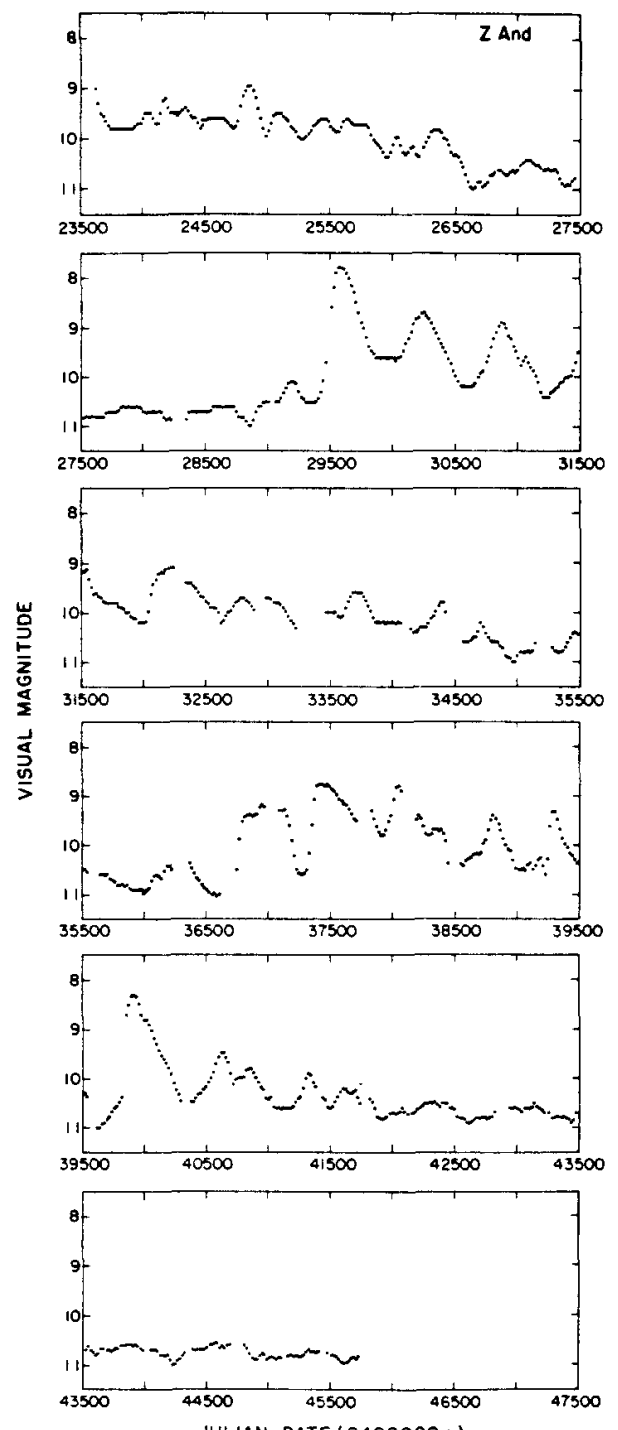

Figure 1 - Optical light curve of $Z$ And as observed by members of the AAVSO (from Kenyon 1986). 


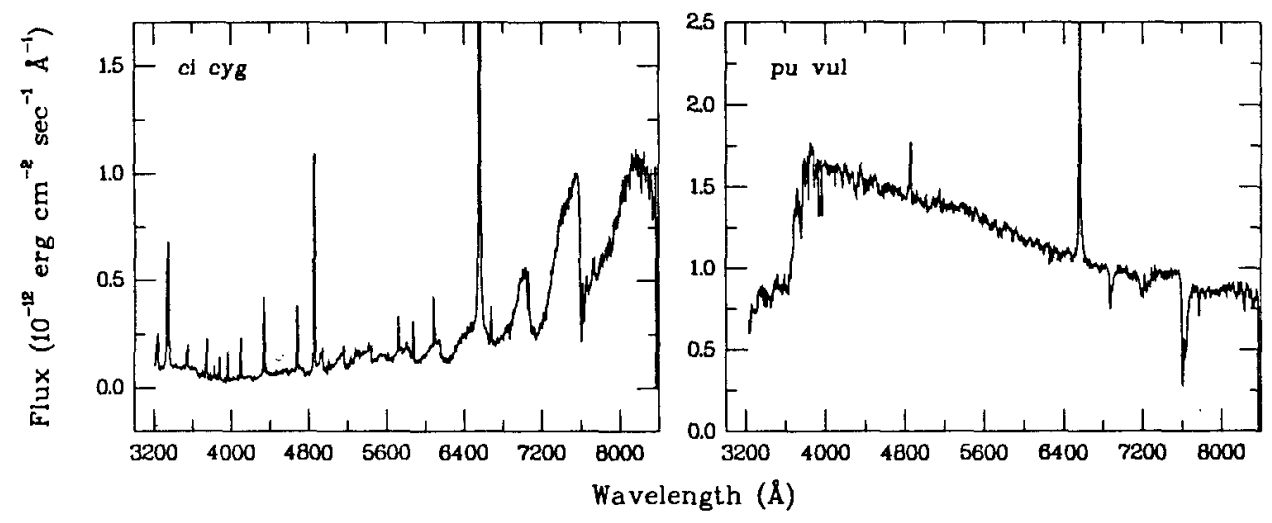

Figure 2 - Optical spectra of CI Cyg and PU Vul obtained at Kitt Peak National Observatory. Strong emission lines (H I, He I, He II, [Ne V], and [Fe VII]) and prominent TiO absorption bands are visible on the quiescent spectrum of $\mathrm{CI}$ Cyg. The eruptive spectrum of PU Vul shows evidence for $\mathrm{H} \mathrm{I}$ emission and the absorption lines characteristic of F stars.

Explanations for the symbiotic phenomenon were debated soon after Merrill and Humason's paper. Both Bcrman (1932) and Hogg (1932) suggested that these objects might be binaries comprised of a rather nomal $M$ star and a hot star similar to the central star of a planctary ncbula. A detailed model for the eruptions of symbiotic stars was not available at this time, but Berman noted that instabilities in the hot object (similar perhaps to those in novae) might give rise to the observed behavior. Kuiper (1941) later introduced Roche geometry into stellar astronomy, and proposed a model for peculiar emission objects in which a star filling its critical Lagrangian surface loses material to its binary companion. Kuiper correctly reasoned that the kinctic energy of infalling matter might give rise to bright emission lines similar to those observed in $\mathrm{AX}$ Per and $\mathrm{T} \mathrm{CrB}$, and noted that the orbital periods deduced for symbiotic stars $\left(P_{\text {orb }}\right.$ - years) required the lobe-filling star to be an $M$ giant. The discovery of radial velocity variations lent some support to these natural binary interpretations for symbiotic stars, although the lack of similar velocity changes in other systems led to the development of rather novel single star mechanisms (see Kenyon 1986).

By the late 1960's, astronomers had achicved an immense amount of descriptive matcrial concerning the behavior of symbiotic stars. It was now well-established that emission lines from a wide range of species, such as $\mathrm{H} \mathrm{I}, \mathrm{He} \mathrm{I,} \mathrm{He} \mathrm{II,} \mathrm{O} \mathrm{III,} \mathrm{Ne} \mathrm{V,} \mathrm{and}$ Fe VII, were typical of many symbiotic stars, and that these lines (and the TiO bands of the giant) fluctuated in intensity on time scales ranging from several days to several years. In spite of this wealth of information, the physical mechanism which causes a star (or stars) to become symbiotic was not identified unambiguously. Advances in technology have led to observations in other wavelength regions and important breakthroughs in understanding the complex symbiotic phenomena observed in the optical. A brief introduction to these data is the subject of this review. 
II. Optical Data and the Defining Features of Symbiotic Stars

An optical spectrum of a fairly normal quiescent symbiotic star, CI Cyg, is shown in the left panel of Figure 2. The characteristic features of the class are readily apparent, and may be briefly summarized:

1. absorption features (TiO, VO, and $\mathrm{Na} \mathrm{I}$ ) observed in red giant stars and an associated red continuum (identified as the cool giant component);

2. strong emission lines from fairly highly ionized species (H I, He I, and [O III]) found in planetary nebulae and a blue continuum (identified as the hot component).

Periodic photometric variations are typical of many systems with amplitudes $\sim 0.5-1.0$ mag and periods of 200-1000 days (see Figure 1). These brightness changes usually are interpreted in terms of orbital motion in a binary system, either as a result of an eclipse of the hot component by the giant, or because the hot star illuminates the facing hemisphere of its giant companion (see Kenyon 1986, Chapter 3).

Aside from the periodic light variations, symbiotic stars display irregular eruptions with amplitudes $-2-7 \mathrm{mag}$. The optical spectrum of an outbursting symbiotic star usually resembles an A-F supergiant, as shown by the spectrum of PU Vul in the right panel of Figure 2. It is obvious that spectral features commonly observed in quiescent symbiotic stars are not present in outburst: Balmer lines are the only emission features. The higher ionization emission lines and the late-type absorption features reappear during the decline from an outburst, and most systems (including the recurrent nova symbiotics $\mathrm{T} \mathrm{CrB}$, RS Oph, and V1017 Sgr) retum to minimum in a few months or a few years. Symbiotic novae require several decades to decline from visual maximum, as discussed by Viotti in this volume.

The motivation for identifying symbiotic stars as binary systems is apparent from inspection of Figure 2. It is fairly natural to associate the absorption features and red continuum with an evolved red giant star and to place the blue continuum and associated emission features with a hot companion star. Direct verification that symbiotic stars are binaries was difficult with the techniques available prior to 1970 , because the small radial velocity amplitudes expected in a binary containing a red giant $\left(-5-10 \mathrm{~km} \mathrm{~s}^{-1}\right)$ cannot be measured easily with photographic plates. Modern observations with photon counting detectors and cross-correlation analysis have demonstrated that all bright symbiotics are binary stars (Garcia, this volume), but estimating physical parameters of the two components require data to supplement optical photometry and spectroscopy. Given the rising red continuum of $\mathrm{Cl}$ Cyg, it makes sense to study the red giant star in the infrared and the information contained in these data is the next topic of discussion.

\section{Infrared Data}

\section{A. Photometry}

Pettit and Nicholson (1933) first demonstrated that infrared (IR) photometry might be a useful probe of the giant components in symbiotic stars with observations of R Aqr. Other symbiotics were too faint to be observed with their bolometer, but modern techniques have shown that most symbiotics appear to be either normal red giants or normal Mira variables when studied at wavelengths of $1-5 \mu \mathrm{m}$. The majority of systems have near-IR colors consistent with stellar photospheric temperatures of $2500 \mathrm{~K}$ to $3500 \mathrm{~K}$, and have been called 

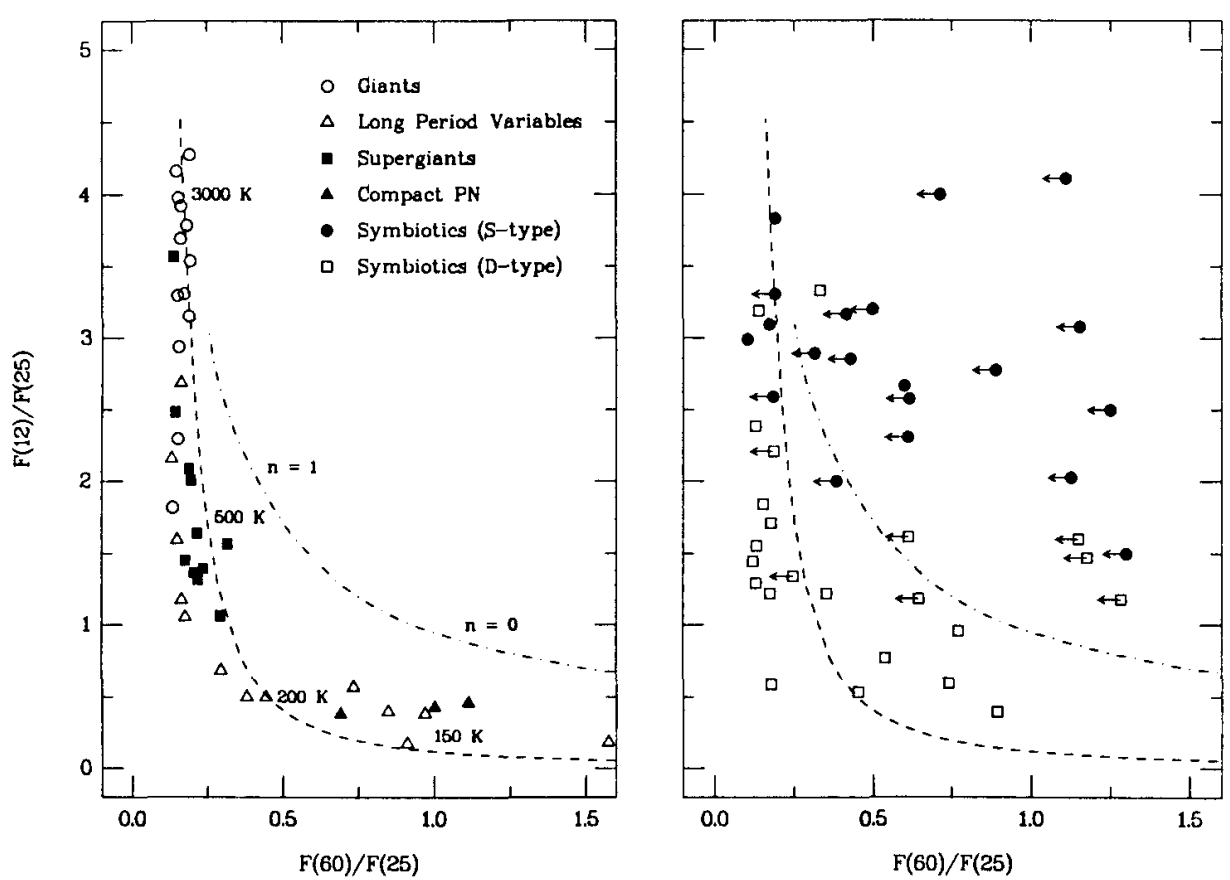

Figure 3 - Flux ratio diagram based on IRAS obscrvations of symbiotics and other M-type stars. The dashed and dot-dashed curves indicate the loci of blackbody and power law sources, respectively (temperatures and power law indices, $F_{v} \propto v^{n}$, are indicate beside each curve). A key for interpreting the symbols is given in the legend. Upper limits to the $60 \mu \mathrm{m}$ flux are indicated by arrows.

S-type (stellar) systems (Webster and Allen 1975). A few other symbiotics have near IR colors more typical of blackbodies with temperatures $\sim 1000 \mathrm{~K}$ and are known as D-type systems (Webster and Allen 1975). These D-type objects have the strong $\mathrm{H}_{2} \mathrm{O}$ absorption bands and periodic variability at $2 \mu \mathrm{m}(\Delta \mathrm{K} \sim 1 \mathrm{mag}, \mathrm{P}-300-600$ days $)$ that typifies Mira variables, although their cncrgy distributions are significantly redder than an average Mira (Allen 1982; Whitelock 1987). Allen (1983) and Kenyon, Fernandez-Castro, and Stencel (1986) showed that the IR continua of several D-type symbiotics could be made to agree with that expected for a Mira if the cool components in these binaries are heavily reddened (extinctions at $\mathrm{K}, \mathrm{A}_{\mathrm{K}} \sim 0.5-2 \mathrm{mag}$ ).

Aside from their near-infrared cmission, symbiotic stars are prodigious far-infrared sources. Over $50 \%$ of known symbiotic stars were detected by the Infrared Astronomical Satellite (IRAS), and a flux ratio diagram based on observed fluxes at 12,25 , and $60 \mu \mathrm{m}$ is presented in Figure 3. It is apparent that the S-type systems are significantly bluer than typical D-type systems in the IRAS survey, although there is some overlap near $F_{v}(12 \mu \mathrm{m}) / F_{v}(25 \mu \mathrm{m})-2$. As might be cxpected from near-IR observations, the S-type systems have far-IR colors similar to those of normal giant stars, while the D-type objects more closcly resemble Mira variables.

The IRAS data are very uscful for cstimating mass loss rates from the cool components of symbiotic stars providing accurate fluxcs are available at 12-60 $\mu \mathrm{m}$ (Whitclock 1987, and this volume; Kenyon, Femandez-Castro, and Stencel 1988). The very large $12 \mu \mathrm{m}$ excesses 


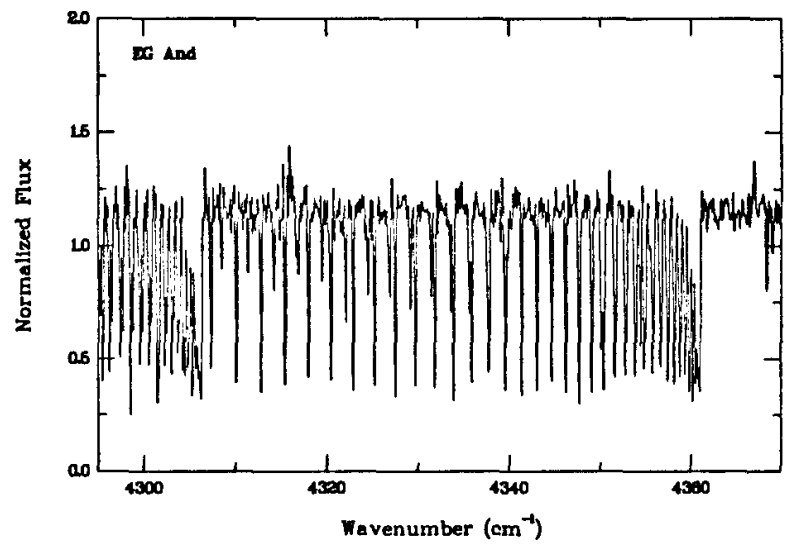

Figure 4 - High resolution IR spectrum of EG And. All of the absorption features in this spectrum are real, and are caused by $\mathrm{CO}$ absorption in the red giant atmosphere.

of the typical D-type object imply mass loss rates, $\dot{\mathrm{M}} \sim 10^{-5} \mathrm{M}_{\odot} \mathrm{yr}^{-1}$, which is somewhat larger than that of most Mira variables (Whitelock, Pottasch, and Feast 1987). Since most S-type systems were not detected by IRAS at $60 \mu \mathrm{m}$, mass loss rates can be estimated only for UV Aur $\left(10^{-6} \mathrm{M}_{\odot} \mathrm{yr}^{-1}\right)$, CH Cyg $\left(6 \times 10^{-8} \mathrm{M}_{\odot} \mathrm{yr}^{-1}\right)$, and AG Peg $\left(2 \times 10^{-7} \mathrm{M}_{\odot} \mathrm{yr}^{-1}\right)$. These mass loss rates are close to those expected for normal M giants (AG Peg and $\mathrm{CH} C \mathrm{Cyg}$ ) and carbon stars (UV Aur).

\section{B. Spectroscopy}

High resolution IR spectra of symbiotic stars have not been obtained as routinely as in the optical or ultraviolet (see below), but significant progress has been made with low resolution $(\lambda / \Delta \lambda \sim 50-200)$ spectrometry. The broad $\mathrm{CO}$ and $\mathrm{H}_{2} \mathrm{O}$ absorption bands present on near-IR spectra of symbiotic stars are typical of $M$ giants and Mira variables, and can be resolved into individual absorption lines with high resolution spectra. An example of the data that can be obtained with the KPNO 4-m Fourier Transform Spectrometer appears in Figure 4. This spectrum of EG And has becn normalized to unity in a standard way (sce Hartmann and Kenyon 1987), but is not flux-calibrated. The strong absorption features are CO lines intrinsic to the $M$ star in EG And, and have a strength comparable to CO lines observed in other M2.5 giants.

Spectroscopic data also have been sccured in the mid-IR with large, ground-based telescopes (Roche, Allen, and Aitken 1983) and with IRAS. Examples of IRAS spectra for three D-type systems are shown in Figure 5, and these data are similar in most respects to mid-IR spectra obtained for very late-type giants and Mira variables. Silicate grain emission at $10 \mu \mathrm{m}$ is very prominent on the IRAS spectra, and is indicative of a large mass loss rate. It appears from the broad band IRAS measurements that symbiotic stars generally display more dust emission than normal red giants of similar spectral types, and their silicate features seem stronger as well.

This brief introduction to the IR observations confirms that the cool components of symbiotic binaries are fairly normal red giant stars, as suggested by optical spectroscopy. 

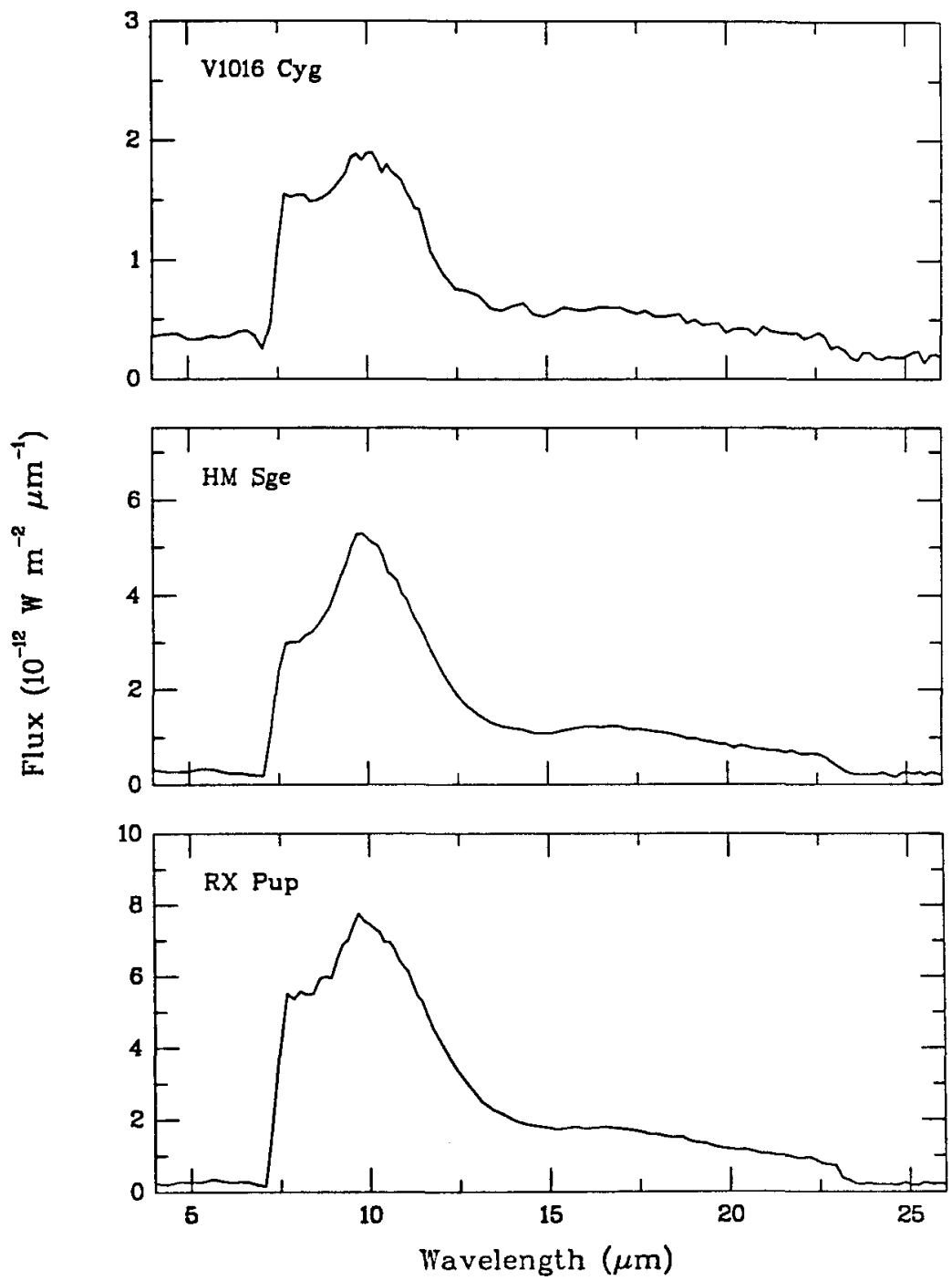

Figure 5 - Low resolution IRAS spectra of several symbiotics.

The division of these objects into S-types and D-types is a major physical result, and will be discussed in more detail later in this volume. Aside from the $\mathrm{H} I$ Paschen and Brackett cmission lines observed in several systems, the IR data reveal little information concerning the physical nature of the hot component. The weak blue continuum present on optical spectra provides one clue that ultraviolet spectroscopy might yield important insights into the energetics of symbiotic stars, and observations obtained in the satellite ultraviolet are the subject of the next section. 


\section{Ultraviolet Spectroscopy}

Initial ultraviolet (UV) observations of symbiotic stars were made by the OAO-2 and TD-1 satellites in distinct photometric bands somewhat analogous to UBVRI optical photometry. The symbiotic nova AG Peg was detected by both UV instruments, and Gallagher et al. (1979) demonstrated that the hot component in this system emitted a continuum similar to that of a Wolf-Rayet star with an effective temperature of $30,000 \mathrm{~K}$. Only one other symbiotic, SY Mus, was detected by TD-1 (and only in two band passes, $1565 \AA$ and $1965 \AA$ ), and OAO-2 failed to detect any other bright symbiotic.

The launch of the International Ultraviolet Explorer (IUE) revolutionized the study of symbiotic stars and demonstrated the validity of the basic binary models proposed in the 1930's and 1940's (see Nussbaumer and Stencel 1987). A spectrum for AG Peg is presented in the left panel of Figure 6, and the very intense blue continuum confirms that the hot component in AG Peg is a stellar source. There is good evidence for the presence of the $2200 \AA$ interstellar absorption feature on this spectrum, and a simultaneous fit for the color temperature of the hot component, $T_{h}$, and the interstellar reddening, $E_{B-V}$, results in $T_{h}=$ $30,000 \mathrm{~K}$ to $60,000 \mathrm{~K}$ and $\mathrm{E}_{\mathrm{B}-\mathrm{v}} \approx 0.11-0.19$ using well-exposed IUE spectra (Kenyon and Webbink 1984).

The majority of symbiotics display UV spectra similar to that shown for AG Peg, and their continua can be understood simply in terms of the Rayleigh-Jeans tail of a hot blackbody and free-bound emission from an ionized nebula. However, several symbiotic stars, as in the spectrum of CI Cyg shown in the right panel of Figure 6, have the fairly flat UV continua more typical of A or B-type stars. It is apparent that an A or B star does not produce sufficient high energy photons to power the emission spectrum, so this simple interpretation of the UV observations cannot be correct. An alternative picture is to suppose such continua arise from an accretion disk surrounding a compact star (see Pringle and Wade 1985). Kenyon and Webbink (1984) showed that the UV data were consistent with this idea only if the accreting star is a low mass main sequence star, and could not identify any symbiotic star with an accreting white dwarf. This model is similar to that proposed by Kuiper (1941) to account for the optical behavior of the same systems.

Emission lines are fairly prominent on UV spectra of symbiotic stars, confirming the presence of the large ionized nebula inferred from optical data. The strongest lines in the spectra usually are from highly ionized species such as He II, C III, C IV, Si IV, and N V, although strong [O I] and $\mathrm{Fe}$ II features have been observed in several instances. Individual emission features have been resolved in the brighter symbiotic objects, and several systems show evidence for gas velocities of $\sim 1000 \mathrm{~km} \mathrm{~s}^{-1}$ (e.g., AG Peg). These velocities are significantly larger than the $-20-100 \mathrm{~km} \mathrm{~s}^{-1}$ expected from material lost by the red giant, and presumably represent gas near the hot component. It is fairly obvious that gas has been ejected at these large velocities in AG Peg, but present observations have not been sufficient to determine if the hot components of other symbiotics are ejecting material or accreting portions of the wind lost by the red giant.

Aside from yielding dynamic information about the ionized gas in symbiotics, high resolution IUE spectra may be used as a probe of the physical conditions in the nebulae (see Nussbaumer and Stencel 1987; Nussbaumer, this volume). Reliable estimatcs for the elcctron density, $\mathrm{n}_{e}$, have been extracted successfully from line ratios of intercombination doublets such as C III] $\lambda \lambda 1907,1909, N$ IV] $\lambda 1483,1487$, and Si III] $\lambda \lambda 1883,1892$, while the O III] and [O III] optical/UV lines provide a useful measure of the electron temperature, $T_{e}$. Typical results for the electron temperature, $\mathrm{T}_{e}-10,000 \mathrm{~K}$ to $20,000 \mathrm{~K}$, suggest that symbiotic nebulae are photoionized rather than shock heated, and the density diagnostics indicate that the gas is much denser, $n_{e} \sim 10^{6}$ to $10^{10} \mathrm{~cm}^{-3}$, than matcrial in typical planetary 


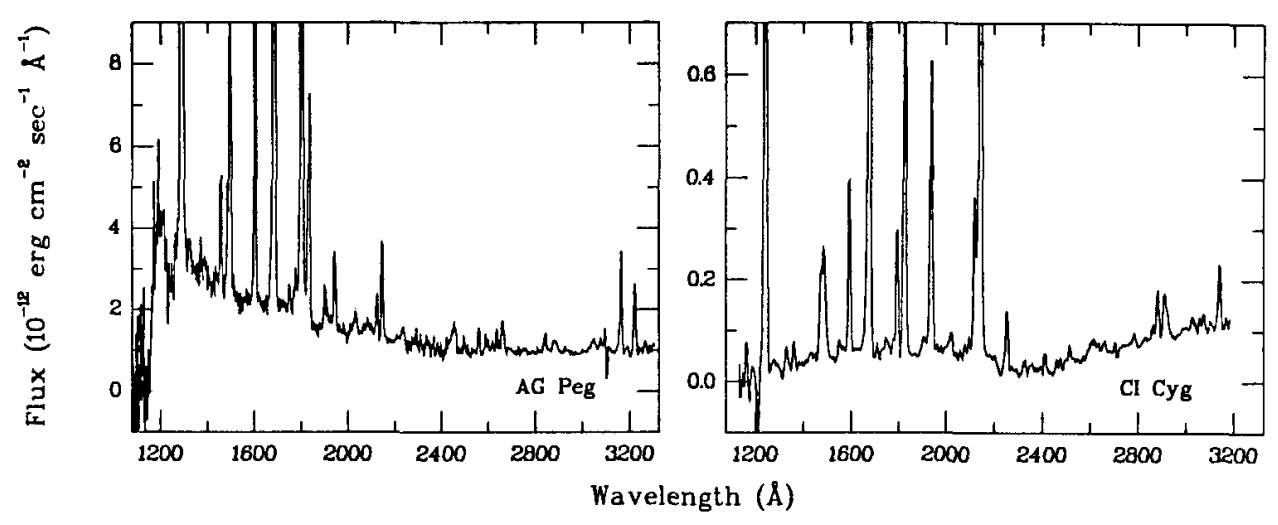

Figure 6 - Ultraviolet spectra for AG Peg (left pancl) and CI Cyg (right panel).

nebulae, $\mathrm{n}_{\mathrm{e}} \leq 10^{4} \mathrm{~cm}^{-3}$.

The UV/optical/IR observations have thus confirmed the natural binary models proposed by Berman, Hogg, and Kuiper when symbiotics were first discovered. By analogy with other interacting binaries, such as cataclysmic variables, symbiotics might be expected to emit soft $\mathrm{X}$-rays as a result of accretion processes. And if the ionized nebula surrounding the binary is sufficiently large, we might anticipate observable amounts of radio emission. Brief summaries of initial $\mathrm{X}$-ray and radio observations of symbiotics are described in the next two sections.

\section{X-rays}

The high blackbody temperatures inferred for several symbiotic stars ( $T_{\text {hot }} \geq 150,000 \mathrm{~K}$ ) suggest that these systems might be soft X-ray sources, but initial searches for X-ray bright symbiotics have been discouraging. Aside from $\mathrm{GX} 1+4$ (which is a neutron star accreting material from the wind of a red giant companion; Doty, Hoffman, and Lewin 1981), symbiotics obscrved with the Einstein satellite were fairly weak sources. Most notable among thesc first detections was the "yellow symbiotic" AG Draconis. Einstein observations obtained before the 1981-1985 series of enuptions recorded $\sim 0.27$ IPC count $\mathrm{s}^{-1}$, as described by Anderson, Cassinelli, and Sanders (1981). These data have been reprocessed, and are consistent with a blackbody source $(\mathrm{kT}=0.016 \pm 0.003 \mathrm{keV}, \mathrm{R}=1500$ $\mathrm{km})$ in addition to the bremsstrahlung source $(\mathrm{kT} \sim 0.1 \mathrm{keV})$ suggested by Anderson, Cassinclli, and Sanders. The X-ray temperature of $\sim 200,000 \mathrm{~K}$ is in fair agrecment with the source temperature of $-100,000 \mathrm{~K}$ to $150,000 \mathrm{~K}$ inferred from $I U E$ observations, although the radius deduced from $I U E$ data, $\mathrm{R}-10^{4} \mathrm{~km}$, is much larger than the $\mathrm{X}$-ray value. Similar disagreements in source parameters result in an analysis of the other Einstein detections, V1016 Cyg and HM Sge, which led to plausible interpretations for the X-rays involving shocks in stellar winds (Kwok and Leahy 1984; Willson et al. 1984).

Several symbiotics were observed by the EXOSAT satellite, which was much more sensitive to soft X-rays than was Einstein. Among the normal symbiotics observed by Einstein, EXOSAT made unambiguous detections of AG Dra and HM Sge, but failed with V1016 Cyg. Observations of R Aqr indicated that high energy photons are associated with 
the jet in this unusual Mira symbiotic, and are consistent with a bremsstrahlung spectrum having $\mathrm{kT}-0.04 \mathrm{keV}$ if the hydrogen column density is fairly low (Viotti $e$ t al. 1987). EXOSAT also detected $\mathrm{CH}$ Cyg following its decline from a recent eruption (Leahy and Taylor 1987).

It is unfortunate that the luminosity and temperature derived from fits to the X-ray data are so very sensitive to the values adopted for the interstellar hydrogen column density, $\mathrm{N}_{\mathrm{H}}$, and the local electron density, $\mathrm{n}_{\mathrm{e}}$. For example, the derived source luminosity for the relatively unreddened symbiotic AG Dra changes by a factor of -150 when the hydrogen column density is increased from $\mathrm{N}_{\mathrm{H}}=0 \mathrm{~cm}^{-3}$ to $\mathrm{N}_{\mathrm{H}}=1.5 \times 10^{20} \mathrm{~cm}^{-3}$ (corresponding to values for the $\mathrm{B}-\mathrm{V}$ color excess of $\mathrm{E}_{\mathrm{B}-\mathrm{V}}=0$ and 0.025 , respectively). The reason for this problem is that symbiotic $X$-ray spectra are very soft (photon energies $<0.1 \mathrm{keV}$ ), and most of the X-rays are efficiently absorbed by interstellar hydrogen and helium atoms along the line of sight.

Finally, it is important to remember that symbiotic stars have significant local hydrogen and helium column densities. For the case of AG Dra, the observed He II $\lambda 4686$ flux indicates $\sim 2 \times 10^{44} \mathrm{He}^{+}$-ionizing photons are absorbed every second by the AG Dra nebula. The luminosity in absorbed $\mathrm{He}^{+}$photons, $\sim 2 \times 10^{34} \mathrm{erg} \mathrm{s}^{-1}$, exceeds the luminosity of the source deduced from the X-ray flux by a factor of two! The degradation of the X-rays by the surrounding nebula thus causes an underestimate in the actual source luminosity, which can be recovered only by an accurate estimate of the soft X-rays absorbed locally. Since V1016 Cyg and HM Sge also have very strong He II $\lambda 4686$ emission lines, the X-rays emitted by these objects are also poorly determined. Thus, a good understanding of symbiotic X-ray spectra awaits better models for their gaseous nebulae.

\section{Radio Emission}

The bright emission lines observed on symbiotic ultraviolet, optical, and infrared spectra suggest the presence of large ionized nebulae surrounding symbiotic binarics, and estimated sizes for the $\mathrm{H}$ II regions are $\mathrm{R}_{\mathrm{H} \text { II }} \sim 1-100 \mathrm{AU}$ (Kenyon 1986). If the ionized gas is optically thick and spherically symmetric, the predicted radio emission at a frequency of $v$ is

$$
S_{v}-0.2\left[\frac{v}{5 \mathrm{GHz}}\right]^{2}\left(\frac{\mathrm{R}_{v}}{\mathrm{~d}}\right)^{2} \mu \mathrm{Jy},
$$

where $R_{v}$ is the radius of the nebula in $A U$ and $d$ is the distance in kpc. Since the current detection limit at the VLA is $\sim 0.1 \mathrm{mJy}$ at $6 \mathrm{~cm}$, only those symbiotics with large radio nebulae, $R_{v}-100 \mathrm{AU}$ for $\mathrm{d} \sim 1 \mathrm{kpc}$, are expected to be radio sources.

Initial detections of symbiotic stars were reported by Wright and Allen (1978; RR Tel), Purton, Feldman, and Marsh (1973; V1016 Cyg), and Altenhoff and Wendker (1973; V1329 Cyg). The most luminous radio source among symbiotics was later found to be $\mathrm{H1}-36\left(\mathrm{~S}_{6} \mathrm{~cm}\right.$ $-45 \mathrm{mJy}$ ), and several other systems have been observed to have radio fluxes $\geq 10 \mathrm{mJy}$ at 6 $\mathrm{cm}$. It is apparent that these symbiotics have very extensive ionized regions, with $\mathrm{R}_{6} \mathrm{~cm} \sim$ 100-1000 AU. As pointed out by Seaquist (this volume), the association of D-type symbiotics with strong radio sources has important consequences for the physical structure of these objects.

Radio maser emission (primarily $\mathrm{SiO}$ and $\mathrm{OH}$ ) is characteristic of many late-type giant stars, but searches for these transitions in symbiotic stars have been discouraging (sce Kenyon 1986). The Mira symbiotic R Aqr is a fairly strong SiO source, but the hot components of other symbiotics appear to be fairly efficient in maintaining conditions that are 
unfavorable for maser emission. There is still some hope that masers might be observed in other systems, because the upper limits reported in the literature remain fairly high. More sensitive searches may be rewarded with new detections.

\section{Summary}

Before this colloquium moves on to a physical interpretation of the symbiotic phenomenon, it is important to note that the basic observational data require symbiotic stars to be long period binary systems. Three components are typically observed:

1. The cool giant component has an effective temperature of $2500-4000 \mathrm{~K}$. In most cases, strong TiO absorption bands and a bright red continuum signify the nature of the giant; these features are not observed on optical spectra of several systems, but infrared $\mathrm{CO}$ and $\mathrm{H}_{2} \mathrm{O}$ bands serve to identify the giant in these instances. IR spectral classification divides the cool components into normal $\mathbf{M}$ giants (S-types) and heavily reddened Mira variables (D-types). Both classes have 10-20 $\mu \mathrm{m}$ excesses signifying large mass loss rates $\left(\sim 10^{-8}\right.$ to $10^{-\top}$ $\mathrm{M}_{\odot} \mathrm{yr}^{-1}$ for S-types; $\sim 10^{-6}$ to $10^{-5} \mathrm{M}_{\odot} \mathrm{yr}^{-1}$ for D-types). However, unlike many evolved stars symbiotics typically do not show evidence for maser emission.

2. The hot companion displays a bright blue continuum at UV wavelengths and sometimes is an X-ray source. Most hot components have UV continua that can be fit by the RayleighJeans tail of a hot $\left(T_{h} \sim 100,000 \mathrm{~K}\right)$, compact $\left(\leq 0.1 \mathrm{R}_{\odot}\right)$ blackbody in combination with nebular continuum radiation. Several objects appear to contain main-sequence stars accreting at rates $\sim 10^{-5} \mathrm{M}_{\odot} \mathrm{yr}^{-1}$.

3. A gaseous nebula envelops the binary. The nebula is denser $n_{c}>10^{6} \mathrm{~cm}^{-3}$ than similar material found in planetary nebulae $\left(\mathrm{n}_{\mathrm{e}}<10^{4} \mathrm{~cm}^{-3}\right)$, and gives rise to substantial radio emission. Local absorption of the $\mathrm{X}$-rays by this nebula probably prevents many symbiotics from being detected by X-ray satellites.

The observations further demonstrate a division into two basic physical classes of interacting binary systems.

1. Detached symbiotics containing a red giant (or a Mira variable) in combination with a hot white dwarf or subdwarf.

2. Semi-detached symbiotics containing a lobe-filling red giant and a solar-type main sequence star.

The hot component accretes material lost by the red giant in both classes: wind accretion dominates the detached systems, while semi-detached objects interact through tidal overflow and the formation of a viscous accretion disk.

The author thanks Mike Garcia for reanalyzing Einstein data for V1016 Cyg, AG Dra, and HM Sge. Portions of this work were supported by NASA through grants NAG5-87 (IUE), 957278 (IRAS Data Analysis Program), and NAG8-605 (HEAO-2 Guest Investigator Program). 


\section{References}

Allen, D.A. 1982. in IAU Colloquium No. 70, The Nature of Symbiotic Stars, ed. M. Friedjung and R. Viotti (Dordrecht: Reidel), p. 27.

Allen, D.A. 1983. Mon. Not. Roy. Astr. Soc., 204, 113.

Altenhoff, W.J. and Wendker, H.J. 1973. Nature, 241, 37.

Anderson, C.M., Cassinelli, J.P., and Sanders, W.T. 1981. Astrophys. J. (Letters), 247, L127.

Berman, L. 1932. Pub. Astr. Soc. Pac., 44, 318.

Doty, J.A., Hoffman, J.A., and Lewin, W.H.G. 1981. Astrophys. J., 243, 257.

Fleming, W.P. 1912. Ann. Harv. Coll. Obs., 56, 165.

Gallagher, J.S., Holm, A.V., Anderson, C.M., and Webbink, R.F. 1979. Astrophys. J., 229, 994.

Greenstein, N.K. 1937. Bull. Harv. Coll. Obs., No. 906.

Hartmann, L., and Kenyon, S.J. 1987. Astrophys. J., 312, 243.

Hogg, F.S. 1932. Pub. Astr. Soc. Pac., 44, 328.

Kenyon, S.J. 1986. The Symbiotic Stars (Cambridge Univ. Press: Cambridge).

Kenyon, S.J., Fernandez-Castro, T., and Stencel, R.E. 1986. Astr. J., 92, 1118.

Kenyon, S.J., Fernandez-Castro, T., and Stencel, R.E. 1988. in preparation.

Kenyon, S.J., and Webbink, R.F. 1984. Astrophys. J., 279, 252.

Kuiper, G.P. 1941. Astrophys. J., 93, 133.

Kwok, S., and Leahy, D.A. 1984. Astrophys. J., 283, 675.

Leahy, D.A., and Taylor, A.R. 1987, Astr. Astrophys., 176, 262.

Lindsay, E.M. 1932. Bull. Harv. Coll. Obs., No. 888.

Merrill, P.W., and Humason, M.L. 1932. Pub. Astr. Soc. Pac., 44, 56.

Nussbaumer, H., and Stencel, R.E. 1987. in Exploring the Universe with the IUE Satellite, ed. Y. Kondo (Dordrecht: Reidel), p. 203.

Payne-Gaposchkin, C. 1957. The Galactic Novae (Amsterdam: North Holland).

Pettit, E., and Nicholson, S.B. 1933. Astrophys. J., 78, 320. Webster, B.L. and Allen, D.A. 1975. Mon. Not. Roy. Astr. Soc., 171, 171.

Purton, C.R., Kwok, S., and Feldman, P.A. 1983. Astr. J., 88, 1825.

Roche, P.F., Allen, D.A., and Aitken, D.K. 1983. Mon. Not. Roy. Astr. Soc., 104, 1009.

Shapley, H. 1922. Bull. Harv. Coll. Obs., No. 778.

Viotti, R., Piro, L., Friedjung, M., and Cassatella, A. 1987. Astrophys. J. (Letters), 319, L7.

Whitelock, P.A. 1987. Pub. Astr. Soc. Pac., in press.

Whitelock, P.A., Pottasch, S.R., and Feast, M.W. 1987. in Late Stages of Stellar Evolution, ed. S. Kwok and S.R. Pottasch (Dordrecht: Rcidel), p. 269.

Willson, L.A., Wallerstein, G., Brugel, E., and Stencel, R.E. 1984. Astr. Astrophys., 133, 154.

Wright, A.E., and Allen, D.A. 1978. Mon. Not. Roy. Astr. Soc., 184, 893. 